\title{
Nicht bloß Falten behandeln, sondern das ganze Gesicht!
}

\author{
Der Begriff „Full-Face-Approach“ bezeichnet ein neues Behandlungskonzept zur Verjüngung des \\ gesamten Gesichts. Es richtet sich nach den individuellen Problemzonen und beinhaltet einen \\ umfassenden Behandlungsplan, der die Bausteine "Relax", "Fill" und "Volumize" mit verschiedenen \\ Produkten umfasst.
}
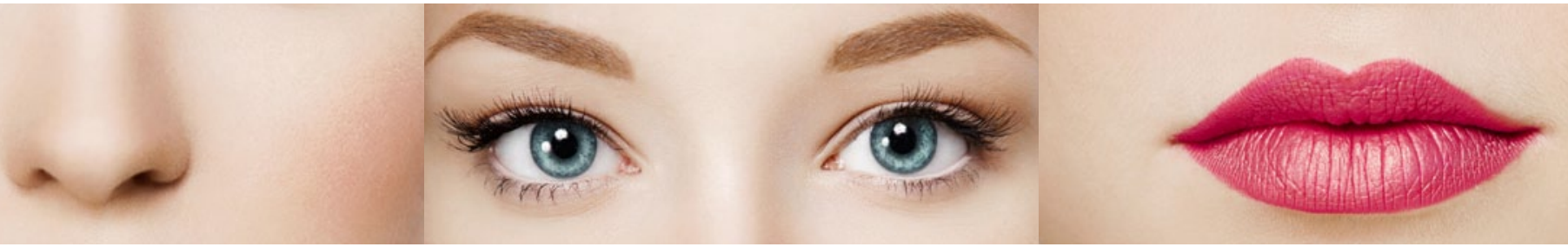

Das ganze Gesicht behandeln - was darunter zu verstehen ist, erläutert Tatjana Pavicic an einem Beispiel. „Wir beobachten tatsächlich, dass Patientinnen beispielsweise wirklich tiefe Glabellafalten aufweisen, das Gesicht hängt nach unten - sie selbst fühlen sich jedoch durch eine kleine, kaum ersichtliche Falte über der Braue gestört“, sagt die Leiterin der Ästhetischen und Laser-Dermatologie an der Dermatologischen Klinik der Münchner Ludwig-Maximilians-Universität. Zwar müsse man die Gefühle des Patienten akzeptieren und ihm zeigen, dass man ihn versteht und auch diese kleine Falte ernst nimmt. Wichtig sei ein offenes und aufklärendes Gespräch. Denn wenn eine wirkliche Verbesserung das Ziel ist, muss laut Pavicic die Behandlung der einzelnen Falte in ein Gesamtkonzept eingebunden werden.

\section{Neue Konzepte erfordern viel Zeit}

„Die neuen Behandlungskonzepte wie der Full-Face-Approach erfordern einfach viel mehr Zeit für Aufklärung“, sagt Pavicic. Denn eine solche Behandlung lasse sich nicht eben mal rasch in der Mittagspause durchführen. Zuerst kommt die Diagnose, bei der festgestellt werden muss, welche Fältchen mimisch bedingt sind, wo Volumendefizite vorliegen und wo die Hautqualität nachgelassen hat. Auch müsse der Patient wissen, "dass er nicht sofort zu einem perfekten Ergebnis kommt“, so die Spezialistin.

„Mit einer Volumenaugmentation streben wir an, jedes Fettkompartiment in seiner originalen Position wieder herzustellen“, erklärt Pavicic. Vor allem im Mittelgesicht geht im Laufe des Alternsprozesses Volumen verloren. Filler, die in dieser Indikation eingesetzt werden, müssen über besondere Eigenschaften verfügen, nämlich hohe Elastizität und Viskosität. Dies hat den Vorteil, dass der Filler dort verbleibt, wo er platziert wird. Hier können sowohl Präparate auf der Basis von Hyaluronsäure mit entsprechenden Eigenschaften eingesetzt werden als auch Filler auf Basis von synthetischen Calcium-Hydroxylapatit-Mikrosphären. Anfängern empfiehlt Pavicic, spitze Nadeln einzu- setzen, weil damit leichter festzustellen ist, wo genau sich die Nadelspitze bzw. das applizierte Produkt befindet. Erst wer genügend Erfahrung gesammelt hat, sollte auf stumpfe Kanülen umsteigen.

\section{Botulinumtoxin fürs obere Drittel}

Der Einsatz von Botulinumtoxin erfordert Muskeln - beim Patienten, nicht beim Therapeuten. Pavicic sagt's lateinisch: „Ubi musculus, ibi Botulinumtoxin." Insofern sei auch wesentlich, zwischen statischen und mimisch bedingten Falten zu unterscheiden. Die in Deutschland verfügbaren Präparate weisen eine vergleichbar gute Wirksamkeit und Verträglichkeit auf. Gefährlich sind Billigimporte aus China - einer Untersuchung zufolge befinden sich häufig andere Dosen in den Produkten als angegeben.

Domäne einer Botulinumtoxin-Behandlung bleibt das obere Gesichtsdrittel. Doch auch im unteren Drittel lassen sich gute Effekte erzielen, allerdings ist hier laut Pavicic der Grat zwischen erwünschter Wirkung und unerwünschten Nebenwirkungen besonders schmal. An der Lippe muss sehr oberflächlich gespritzt werden, die Dosis ist entscheidend, und speziell hier ist „weniger oft mehr“. Die Pars labialis des M. orbicularis oris zieht die Lippe nach innen und führt so zu einer optischen Verkleinerung. Durch die Behandlung dieses Muskelanteils mit Botulinumtoxin kommt es zur Eversion der Lippe. Dadurch wird optisch ein größeres Lippenvolumen erreicht. An diese Indikation sollten sich aber nur fortgeschrittene Anwender wagen. Patienten, die den vollen Einsatz der Stimme benötigen, wie Moderatoren oder Sänger, sollten in der Mundregion grundsätzlich nicht mit BTX-A behandelt werden.

Bei den einzelnen Behandlungsterminen sollte man nicht $\mathrm{zu}$ viel auf einmal in Angriff nehmen. Empfehlenswert ist es, sich im Therapieplan etappenweise Ziele zu stecken. Dies führt zu einer höheren Patientenzufriedenheit als eine zu starke bzw. schnelle und plötzliche, auffallende Veränderung, die dann auch von der Mitwelt bemerkt wird. 